\title{
Inventario de talento informático en GADE y GGAP
}

\author{
Baviera, M. Amparo; Babiloni, Eugenia; Debón, Ana; Marin, $M^{a}$ del Mar; Puertas, Rosa; Ribal, \\ Javier; Skorczynska, Hanna Teresa y Vallada, Eva \\ ${ }^{a}$ Facultad de Administración y Dirección de Empresas. Universitat Politécnica de València
}

ambapui@upv.es; mabagri@doe.upv.es; andeau@eio.upv.es; mmarins@esp.upv.es; rpuertas@esp.upv.es; frarisan@esp.upv.es; hskorczy@idm.upv.es; evallada@eio.upv.es

\begin{abstract}
Universities must provide enough skills to their students so as to favor the access to the labor market.. The aim is to provide not only technical knowledge but also to introduce competencies that complete the training in the learning process, adjusting the curricular programs to the private sector demands.

In this context, the Universitat Politècnica de Valencia and specifically, its Faculty of Business Administration and Management, has decided to opt for the accreditation of IT and ICT-related skills that can be obtained through its degrees in Business Administration and Management, and Public Administration and Management

The main achievement of this project is that our students will have a differentiating aspect in their file compared to other degrees from other centers. The fact of being at the Polytechnic University of Valencia means that our degree students are always in contact with the technology and the appropriate computer tools for each subject. This project helps visualize this aspect both for students and employers.
\end{abstract}

Keywords: Soft skills, Business Administration, Public Administration, Software.

\section{Resumen}

La Universidad debe proporcionar a los egresados las habilidades necesarias para su adecuada introducción en el mercado laboral. Se trata de aportar no solo conocimientos técnicos sino también implantar en el proceso de aprendizaje competencias que completen la formación, adecuando los programas curriculares a las exigencias del sector privado.

En este contexto, la Universitat Politècnica de Valencia y en concreto, su Facultad de Administración y Dirección de empresas ha decidido apostar por la acreditación de habilidades informáticas y relacionadas con las TIC que pueden obtenerse mediante sus grados: en Administración y dirección de Empresas , y Gestion y Administracion Pública.

El principal logro del presente proyecto es que nuestros estudiantes contarán con un aspecto diferenciador en su expediente respecto a títulos de otros centros. El hecho de estar en la Universitat Politècnica de València hace que nuestros estudiantes de grado estén en contacto continuamente con la tecnología y las herramientas informáticas adecuadas para cada asignatura, y este proyecto visualiza dicho aspecto para el estudiante y el empleador

Palabras clave: Competencias transversales, Grado de ADE, Grado de GAP, Software. 


\section{Introducción}

La evolución del sistema educativo en todos sus niveles está en un momento de inflexión donde la adquisición de conocimientos, destrezas, actitudes y valores se entremezclan en las aulas para facilitar la aplicación de lo aprendido a situaciones muy diversas. Así pues, el objetivo formativo de la Universidad debe consistir en proporcionar a los egresados las habilidades necesarias para su adecuada introducción en el mercado laboral. Se trata de aportar no solo conocimientos técnicos sino también implantar en el proceso de aprendizaje competencias que completen la formación, adecuando los programas curriculares a las exigencias del sector privado. Según Ramírez (2010), la capacitación por competencias enfatiza la adquisición de habilidades prácticas para su desempeño óptimo en un contexto laboral, económico y académico en busca de una óptima empleabilidad. Sin embargo, autores como Bassi et al (2012), Cappelli (2014) o Mourshad et al (2011), entre otros, aportan evidencias del diferencial existente entre las habilidades laborales ofertadas y la demanda existente en el entramado productivo, limitando la capacidad de poder acceder a trabajos de calidad.

Los certificados de notas aportan al empleador información sobre el nivel de conocimientos específicos de los candidatos a ocupar determinados puestos de trabajo, existiendo un vacío en torno a las competencias transversales (CT). Por tanto, y de forma adicional, será necesario disponer de una valoración institucional de las CT de los egresados que aspiren a ocupar un puesto en el mercado laboral. La Universitat Politècnica de València (UPV) se halla inmersa en un proceso de adaptación para acreditar las habilidades de sus egresados, estableciendo sinergias con las organizaciones productivas, para adaptarse a las exigencias del mercado.

Normalmente existe una confusión entre los términos "certificación" y "validación". Según Bertrand (2000) la certificación es el proceso por el cuál se asegura que un individuo ha alcanzado un determinado nivel de conocimiento, habilidades o capacidad de aprendizaje de una determinada materia. Se trata de un reconocimiento de competencias obtenidas a través de un sistema de aprendizaje formalizado, estando la validación asociada a logros menos normalizados. Por su parte, Medina (2006) mezcla ambos conceptos e indica que la acreditación se refiere a la evaluación de conocimientos y experiencias adquiridos mediante aprendizajes formales y/o informales cuyo nivel se refleja mediante la obtención de un título, es decir, una certificación.

Actualmente, casi la totalidad de los alumnos universitarios son nativos digitales, definidos por Sánchez y Castro (2013) como aquellos jóvenes nacidos en la era del desarrollo masivo de la tecnología de la información y la comunicación (TIC), con acceso a herramientas informáticas que utilizan y manejan con gran destreza. Las distintas materias que componen los planes docentes de los actuales grados introducen, en el desarrollo del contenido práctico de cada asignatura, el manejo de determinados programas informáticos como herramienta de apoyo para la consecución de fines específicos de diversa índole académica: economía, matemáticas, estadística, finanzas, entre otras. Se trata de potentes instrumentos que facilitan la trasmisión de conocimientos y aventuran al alumno en técnicas digitales de gran ayuda para su completa capacitación, que no finaliza en ellos mismos, sino que precisan de una continua actualización.

El mundo digital y las necesidades operativas evolucionan con gran rapidez, muy probablemente los conocimientos aprendidos en las aulas estén obsoletos cuando los alumnos finalicen sus estudios. Por ello es importante, por parte del docente, facultar al estudiante para un aprendizaje autodidacta y adaptativo, capacidades que faciliten la formación continua y la acomodación a los cambios que, con toda seguridad, se irán sucediendo. 
En este sentido, Oliver et al. (2000) señalan el esfuerzo que supone definir un conjunto de habilidades que describan adecuadamente a una persona con conocimientos de informática así como la necesidad de diseñar herramientas de evaluación para medir los niveles de estas habilidades, una tarea difícil en un entorno tan multifacético. Tomando en este trabajo la definición ampliamente aceptada de alfabetización informática (“computer literacy") como la de Simonson et al (1987) quienes definen la alfabetización informática como:

"Una comprensión de las características, capacidades y aplicaciones de la computadora, así como la capacidad de implementar este conocimiento en el uso hábil y productivo de aplicaciones informáticas adecuadas para roles individuales en la sociedad. (pág. 232)".

El concepto de certificación es la confirmación de ciertas características de un objeto, persona u organización. Suele ser otorgada por algún tipo de revisión, valoración o auditoría externa. Se habla de acreditación cuando es el proceso de certificación específico de una organización. La certificación en una herramienta informática valida las habilidades profesionales que garantizan el dominio de nuevas competencias.

La acreditación de habilidades informáticas y relacionadas con las TIC puede obtenerse mediante títulos específicos de los programas normalmente vinculados a empresas comerciales fabricantes del software. En este sentido, destacan las certificaciones de Microsoft (MOS, Microsoft Office Specialist). Un segunda forma es conseguir un documento que acredite los conocimientos asociados a la utilización de herramientas informáticas de modo más genérico, no vinculado a un programa específico si no a una tipología, por ejemplo, la ECDL (European Computer Driving Licence). Se trata de una acreditación internacional europea que otorga el reconocimiento de poseer una formación básica y completa en informática a nivel de usuario, estando implantada en toda Europa y en el resto del mundo, bajo las siglas ECDL. ECDEL define tres niveles de certificación basados en la consecución de un serie de módulos, de menor a mayor nivel (Esencial, Extra y Core). Todos ellos tratan sobre fundamentos, procesador de textos, hojas de cálculo, bases de datos, software de presentaciones, internet y productividad.

En España, existe el proyecto CertiUni que tiene como finalidad promover la cultura de la certificación personal, ofreciendo sistemas de acreditación a las Universidades y Empresas en algunas de las competencias más demandadas en el nuevo Espacio Europeo de Educación Superior. CertiUni fue promovido por la Conferencia de Rectores de las Universidades Españolas (CRUE), ofrece certificaciones diseñadas por diferentes empresas del sector (Microsoft, Oracle, Adobe, etc) ligadas a programas comerciales concretos.

Atendiendo a su certificación, Andino y Caballero (2003) describen dos programas de certificación empresarial en el ámbito de aplicaciones informáticas. Uno más comercial, el ya citado Microsoft Office Specialist, en el que se detectan innumerables deficiencias como el coste del examen, ausencia de requisito académico, falta de utilidad en el mercado laboral, entre otras. $\mathrm{Y}$ otro propuesto por la Universidad Autónoma de la Laguna (UAL) en el marco de un convenio con la empresa IBM, UAL-IBM, para incorporar certificaciones de IBM en temas de comercio electrónico en el Grado de Ingeniería en Sistemas Computacionales (ISC). Este programa está diseñado para tener una duración de 464 horas de duración desarrolladas a lo largo de sus estudios, de manera que, al finalizar el grado, además del título universitario correspondiente, los egresados obtienen la certificación de IBM como "Desarrolladores de Aplicaciones de Comercio Electrónico".

Tobón (2010) considera que la certificación de competencias es clave en la educación superior. Se requiere disponer de mecanismos sólidos para que tenga valor no sólo en el ámbito laboral y profesional, sino también en el contexto educativo. Ello facilitaría la flexibilidad de los itinerarios educativos, 
pudiendo acceder a determinados ciclos acreditando la posesión de las competencias previas requeridas. Con objeto de otorgar imparcialidad y objetividad al nivel competencial alcanzado y acreditado en el certificado, Tobón propone la creación de instituciones certificadoras ajenas a la institución universitaria.

Gutiérrez et al. (2017) proponen un instrumento para evaluar las competencias digitales del alumnado universitario. También es importante conocer la percepción de los estudiantes sobre su nivel de competencia digital (Hernández y San Nicolás, 2019).

El concepto de certificación es la confirmación de ciertas características de un objeto, persona u organización, otorgada por algún tipo de revisión, valoración o auditoría externa. Se trata de acreditación cuando es el proceso de certificación específico de una organización. La certificación en una herramienta informática valida las habilidades profesionales con dicha herramienta y garantizan el dominio de nuevas competencias asociadas a ella.

En el ámbito que compete al proyecto TALiFADE que agrupa la certificación en sus dos grados (TALiADE y TALiGAP) irá vinculada a la emisión de un documento por parte de la Facultad de Administración y Dirección de Empresas (FADE) de la UPV. En él se concretarán las destrezas informáticas adquiridas por los alumnos en el desarrollo de las distintas asignaturas que definen el plan de estudios de los dos grados impartidos en esta facultad: grado en Administración y Dirección de Empresas (GADE) y grado en Gestión y Administración Pública (GGAP). Ahora bien, la emisión de este certificado requiere la definición de un proceso de evaluación continua de las mismas durante todo el proceso de formación universitaria.

\section{Objetivos}

La UPV y, particularmente, el Instituto de Ciencias de la Educación ha apostado por una enseñanza basada en competencias, desarrollando un proyecto común para todas las titulaciones impartidas en ella. Se han definido 13 Competencias Transversales introduciéndolas en todos los grados y másteres, siendo necesario el aprendizaje y evaluación continua de todas ellas.

Concretamente, el proyecto institucional TALiFADE se articula sobre la competencia 13, "Instrumental específica", entendida ésta como la capacidad de utilizar las técnicas, habilidades y herramientas actualizadas necesarias para la práctica de la profesión. Esta competencia hace referencia al uso de las tecnologías necesarias para el ejercicio profesional asociado a cada titulación. El estudiante debe ser capaz de identificar las herramientas más adecuadas en cada caso, conociendo sus utilidades. Además, debe adquirir el conocimiento necesario que le permita integrarlas y combinarlas para poder resolver un problema, realizar un proyecto o un experimento.

En primer lugar, se dotará de contenido a la competencia transversal 13 en GADE y en GGAP, realizando un inventario del talento informático de nuestros titulados que será recogido en el expediente académico. De esta forma se hará visible para el empleador como un atributo diferencial de nuestros estudiantes.

En segundo lugar, se persigue aumentar las capacidades informáticas de los egresados de GADE y GGAP, estableciendo los siguientes objetivos específicos:

O1. Inventariar el uso de programas informáticos en la titulación.

O2. Definir itinerarios de asignaturas para cada programa informático.

O3. Lograr una conexión interdisciplinar de cada herramienta informática utilizada. Se realizará durante el segundo año.

O4. Creación de rúbricas de evaluación de la competencia transversal 13 para cada itinerario. Se alcanzará durante el segundo año. 
O5. Certificar e integrar los conocimientos de cada software en el expediente académico del estudiante. Se alcanzará durante el segundo año.

\section{Desarrollo de la innovación}

En lo referente al desarrollo de la innovación docente se ha iniciado formando un grupo multiexperto procedente de la titulación GADE y GGAP caracterizado por las siguientes capacidades:

- Experiencia docente amplia en las asignaturas de la titulación.

- Sólida base en programas informáticos de cada asignatura.

- Amplios conocimientos del Poliformat y, en particular, en la generación de baterías de preguntas y exámenes.

- Adecuada formación de los docentes en la competencia instrumental específica, que permita la correcta formación y evaluación de los alumnos.

Para poder cubrir con adecuación a las tres primeras capacidades se han seleccionado profesores expertos en cada asignatura de las titulaciones. En cuanto a la cuarta capacidad, los cursos de formación del ICE y el propio esfuerzo del profesorado implicado le ha habilitado para el correcto desarrollo de la misma.

El proyecto se divide en tres fases, todas ellas estarán coordinadas por las directoras académicas de los respectivos grados, Eva Vallada Regalado y Rosa Puertas Medina .

En la Fase I se vertebra la organización tanto de información como de personas. La propia Facultad, específicamente su equipo directivo, participa de forma activa junto con la responsable del proyecto. En la segunda fase, Desarrollo y Aplicación, las unidades básicas serán equipos de docentes, formados en la Fase I, con un coordinador por equipo. La Fase III incluye el diseño de la acreditación del talento informático.

A continuación, se detallan las tareas a realizar en cada fase, su relación con los objetivos, así como su responsable. La Figura 1 muestra el conjunto del plan de trabajo.

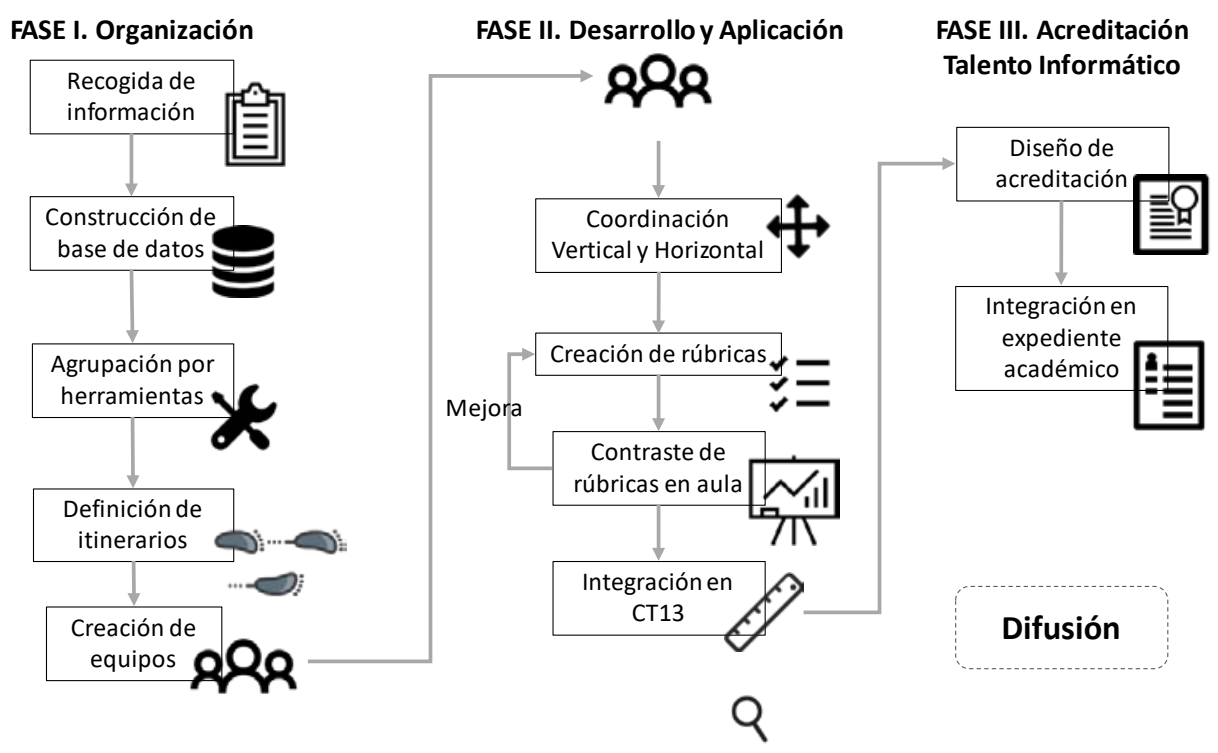

Figura 1. Fases y tareas del plan de trabajo. 


\subsection{Formulario}

En primer lugar, se ha elaborado un formulario, a través de la herramienta Google FormS, para recopilar información sobre los programas informáticos utilizados en cada asignatura de las titulaciones de GADE y GGAP $^{1}$, y se ha enviado a los profesores que imparten las distintas asignaturas. En el formulario el profesor selecciona la asignatura y especifica el programa informático utilizado, así como las horas dedicadas a ello. El formulario se ha enviado durante el primer año de realización del presente proyecto. Con esa información se ha construido la base de datos.

\subsection{Construcción de base de datos}

La base de datos almacena una serie de variables que serán fundamentales a la hora de extraer los primeros resultados. Dicha base de datos es un fichero Excel donde se recoge: titulación, nombre de asignatura, software utilizado, número de créditos.

Esta base de datos ha permitido obtener los primeros resultados que se concretan en la definición de itinerarios y formación de grupos de trabajo. Además ha facilitado la cuantificación de las horas que dedican los estudiantes a cada software y su seguimiento a lo largo de los cursos.

\section{Resultados}

La información recogida a través del formulario ha sido integrada en el plan de estudios, materias y POD, construyendo una base de datos. A partir de ella se ha elaborado un informe de inventario de uso de programas informáticos en cada grado.

\subsection{Resultados para GADE}

En GADE se han definido 3 itinerarios de asignaturas que utilizan el mismo programa informático: Excel, Estadística y Ofimatica (Tabla 1, 2 y 3, respectivamente). Las columnas que definen estas tablas (Tabla 1, 2 y 3) indican el nombre y código de la asignatura, el software ( $\mathrm{Sw}$ ), el curso y si es considerada obligatoria en el plan de estudio (Oblig).

A continuación, se han creado equipos de trabajo formados por profesores de distintas asignaturas que utilizan el mismo programa informático, nombrando un coordinador en cada uno de ellos.

Cada responsable se encargará de reunir a los profesores de las asignaturas correspondientes al programa informático con el objetivo de crear una serie de ítems para la evaluación de los conocimientos de dicho programa.

\footnotetext{
${ }^{1}$ disponible en https://docs.google.com/forms/d/1T_110jQIprI1nDYAYyGefd8tHmWwXA6viwhUlh079Ek/edit
} 
Tabla 1: Itinerario Excel en GADE

\begin{tabular}{llcc}
\hline Nombre Asignatura & Sw & Curso & Oblig \\
\hline Introducción a las Finanzas (11737) & Excel & $1 \mathrm{~A}$ & Sí \\
Microeconomía I (11738) & Excel & $1 \mathrm{~A}$ & Sí \\
Matemáticas Financieras (11750) & Excel & 1B & Sí \\
Microeconomía II (11759) & Excel & 1B & Sí \\
Contabilidad Financiera y de Sociedades (11746) & Excel & 2A & Sí \\
Dirección de Producción y Operaciones (11748) & Excel & $2 \mathrm{~A}$ & Sí \\
Economía Española (11758) & Excel & $2 \mathrm{~B}$ & Sí \\
Estrategia y Diseño de la Organización (11747) & Excel & $2 \mathrm{~B}$ & Sí \\
Contabilidad de Costes e Introducción a la Auditoría (11745) & Excel & $3 \mathrm{~A}$ & Sí \\
Investigación Operativa (11761) & Excel & $3 \mathrm{~A}$ & Sí \\
Análisis y Consolidación Contable (11744) & Excel & $3 \mathrm{~B}$ & Sí \\
Economía Financiera (11752) & Excel & $3 \mathrm{~B}$ & Sí \\
Planes estratégicos en las empresas (11769) & Excel & $3 \mathrm{~B}$ & No \\
Banca y Bolsa (11774) & Excel & $4 \mathrm{~A}$ & No \\
Dirección de Recursos Humanos (11749) & Excel, & 4A & Sí \\
Dirección Financiera (11751) & Word & & \\
Logística (11793) & Excel & $4 \mathrm{~A}$ & Sí \\
Valoración de empresas (11776) & Excel & $4 \mathrm{~B}$ & No \\
\hline Fuente: Elaboración Propia & Excel & $4 \mathrm{~B}$ & No \\
\hline
\end{tabular}

Tabla 2: Itinerario Estadística en GADE

\begin{tabular}{|c|c|c|c|}
\hline Nombre Asignatura & Sw & Curso & Oblig. \\
\hline Econometría (11762) (Grupo ARA) & $\mathrm{R}$ & $2 \mathrm{~B}$ & Sí \\
\hline Métodos Estadísticos en Economía (11741) & $\mathrm{R}$ & $2 \mathrm{~A}$ & Sí \\
\hline Econometría (11762) (excepto grupo ARA) & Gretl & 2B & Sí \\
\hline Gestión de Calidad (11770) & Statgraphics & $4 \mathrm{~A}$ & No \\
\hline Introducción a la Estadística (11740) & Statgraphics & $1 \mathrm{~B}$ & Sí \\
\hline
\end{tabular}

Fuente: Elaboración Propia

Tabla 3: Itinerario Ofimática en el GADE

\begin{tabular}{llll}
\hline \multicolumn{1}{c}{ Nombre Asignatura } & \multicolumn{1}{c}{ Sw } & Curso & Oblig. \\
\hline Introducción a la Administración de Empresas (11735) & Ofimática & 1A & Sí \\
Derecho de la Empresa (11742) & Word & 1A & Sí \\
Microeconomía II (11759) & Powerpoint & 1B & Sí \\
Estrategia y Diseño de la Organización (11747) & Word & 2B & Sí \\
Análisis y Consolidación Contable (11744) & Word & 3B & Sí \\
Planes estratégicos en las empresas (11769) & Powerpoint & 3B & No \\
\hline \multicolumn{1}{c}{ Fuente: Elaboración Propia } & & &
\end{tabular}

\subsection{Resultados para GGAP}

Las particularidades propias de este grado, con más carga legislativa, ha permitido definir tan sólo łos 2 itinerarios de asignaturas que utilizan el mismo programa informático: Ofimatica y Estadística (Tablas 4 y 5 , respectivamente). Las columnas que definen estas tablas (Tablas 4 y 5) indican el nombre y código de la asignatura, el software ( $\mathrm{Sw}$ ), el curso y si es considerada obligatoria en el plan de estudio (Oblig). 
Tabla 4: Itinerario Ofimática en GGAP

\begin{tabular}{|c|c|c|c|}
\hline Nombre Asignatura & Sw & Curso & Oblig \\
\hline Informática aplicada (10601) & $\begin{array}{l}\text { Open } \\
\text { Office }\end{array}$ & $1 \mathrm{~A}$ & Sí \\
\hline Gestión Financiera (10612) & Excel & $2 \mathrm{~A}$ & No \\
\hline Teoría de organizaciones (10596) & Office & $2 \mathrm{~A}$ & Sí \\
\hline Técincas y Métodos de Gestión Públicas (10606) & Excel & $2 \mathrm{~B}$ & Sí \\
\hline Gestión de Recursos Humanos (10618) & $\begin{array}{l}\text { Word } \\
\text { Excel }\end{array}$ & $3 A$ & Sí \\
\hline Gestión Presupuestaria (10613) & Excel & $3 A$ & Sí \\
\hline $\begin{array}{l}\text { Control de Costesen los sistemas de salud y servicios } \\
\text { sociales públicos (10621) }\end{array}$ & Excel & $4 \mathrm{~B}$ & No \\
\hline Gestión de la Política Industrial y tecnológica (10624) & $\begin{array}{l}\text { Excel } \\
\text { Word }\end{array}$ & $4 \mathrm{~B}$ & No \\
\hline $\begin{array}{l}\text { Auditoría de los Sistemas de Información en Organizaciones } \\
\text { Públicas (10622) }\end{array}$ & Word & $4 \mathrm{~B}$ & No \\
\hline Inglés para la Administración (10661) & Word & $4 \mathrm{~A}$ & No \\
\hline
\end{tabular}

Tabla 5: Itinerario Estadística en GGAP

\begin{tabular}{llll}
\hline Nombre Asignatura & Sw & Curso & Oblig. \\
\hline Introducción a la Estadística (10598) & Statgraphics & 2A & Sí \\
Estadística Aplicada a la Administración Pública (10607) & R & 2B & Sí \\
Maketing en el Sector Público (10625) & Gretl & 2B & Sí \\
\hline Fuente: Elaboración Propia & & &
\end{tabular}

Igualmente, se han creado equipos de trabajo formados por profesores de las asignaturas que utilizan el mismo programa informático, nombrando un coordinador de cada uno de ellos.

Estos responsables se encargarán de reunir a los profesores de las asignaturas correspondientes al programa informático, con objeto de crear una serie de ítems para la evaluación de los conocimientos de dicho programa.

Durante el segundo año de proyecto se han formado los equipos de trabajo, los coordinadores están realizando reuniones con los profesores implicados y elaborando las rúbricas para su posterior uso en las aulas con el objetivo de evaluar el nivel alcanzado en las herramientas informáticas.

Finalmente se está estudiando cómo diseñar una acreditación y su integración en el expediente académico de los estudiantes, a través de la competencia transversal 13, Instrumental específica.

\section{Conclusiones}

El principal logro del presente proyecto es que nuestros estudiantes contarán con un aspecto diferenciador en su expediente respecto a títulos de GADE y GGAP impartidos por otros centros. Las características propias de la UPV facilitan que los estudiantes de GADE y GGAP estén en contacto continuo con la tecnología y las herramientas informáticas adecuadas para cada asignatura. Este proyecto trata de dar visibilidad a este aspecto tanto para el estudiante como para el empleador.

Otro aspecto importante está siendo la coordinación entre asignaturas que utilizan un mismo programa informático, permitiendo constatar el avance de los alumnos y evitando solapamientos que impidan su desarrollo gradual. Las reuniones periódicas de los profesores están permitiendo establecer una ruta para alcanzar los conocimientos necesarios de las aplicaciones informáticas. 
Sin embargo, y a pesar de que a nivel global la cooperación de los profesores implicados está siendo adecuada, todavía existen algunas reticencias a introducir cambios metodológicos que pudieran alterar el desarrollo cotidiano de la docencia.

\section{Referencias}

ANDINO, M. y CABALLERO, J. (2003). "La alianza universidad-empresa en la certificación de conocimientos y habilidades: el caso de la Universidad Autónoma de la Laguna". E-Gnosis, issue 1, art.5.

BASSI, M., BUSSO, M. URZÚA, S. y VARGAS, J. (2012). Desconectados: Habilidades, educación y empleo en América Latina. Washington: Banco Interamericano de Desarrollo.

BERTRAND, O. y IBERFOP (2000). "Profesional. Evaluación y certificación de competencias y cualificaciones profesionales". Programa de Cooperacion Iberoamericana para el Diseño de la Formacion. Cooperación Iberoamericana. Madrid

CAPPELI, P. (2014). "Skill gaps, skill shortages and skill mismatches: evidence for the US". NBER Working Paper No 20382

GUTIÉRREZ, J.J., CABERO, J., ESTRADA, L.I. (2017). "Diseño y validación de un instrumento de evaluación de la competencia digital del estudiante universitario". Revista espacios, vol. 38, issue 7, p. 1-27.

HERNÁNDEZ-RIVERO, V.M., SAN NICOLÁS-SANTOS, M.B. (2019). "Percepción del alumnado universitario sobre su grado de competencia digital". Hamut'ay, vol. 6, issue 1, p. 7-18.

MEDINA, O. (2006). "Los sistemas de acreditación. Aproximación conceptual y teórica". Educar, issue 38, p. $105-131$

MOURSHAD, M., FARRELL, D. y BARTON, D. (2011). From Education to Employment: Designing a System that Works. New York: McKinsey and Company.

OLIVER, R., TOWERS, S., \& OLIVER, H. (2000). "Information and Communications Technology LiteracyGetting serious about IT." In EdMedia+ Innovate Learning). Association for the Advancement of Computing in Education (AACE). p. 862-867.

RAMÍREZ, A. (2010). Educational video: Exploring the complex relationship between production, educational use and audience. Tesis doctoral. Universidad de Lancaster (Inglaterra)

SÁNCHEZ-ESPINOZA, A, y CASTRO-RICALDE, D. (2013). "Cerrando la brecha entre nativos e inmigrantes digitales a través de las competencias informáticas e informacionales". Apertura, vol. 13, issue 19, p. 6-15

SIMONSON, M. R., MAURER, M., MONTAG-TORARDI, M., \& WHITAKER, M. (1987). "Development of a standardized test of computer literacy and a computer anxiety index". Journal of educational computing research, 3(2), 231-247.

TOBÓN, S. (2007). "El enfoque complejo de las competencias y el diseño curricular por ciclos propedéuticos". Acción Pedagógica, vol. 16, issue 1, p. 14-28 